\title{
Multidisciplinary management of temporomandibular joint ankylosis in an adult: journey from arthroplasty to oral rehabilitation
}

\author{
Kamlesh Kothari, ${ }^{1}$ Naveenkumar Jayakumar (D) , ${ }^{2}$ Aayesha Razzaque ${ }^{1}$
}

${ }^{1}$ Oral \& Maxillofacial Surgery, Aesthetica, Kolkatta, India

${ }^{2}$ Head - Oral and Maxillofacial Surgery, Sri Ramachandra Institute of Higher Education and Research, Chennai, India

\section{Correspondence to}

Professor Naveenkumar Jayakumar;

naveenkumarj@sriramachandra. edu.in

Accepted 27 August 2021

Check for updates

(c) BMJ Publishing Group Limited 2021. No commercial re-use. See rights and permissions. Published by BMJ.

To cite: Kothari $\mathrm{K}$

Jayakumar N,

Razzaque A. BMJ Case

Rep 2021;14:e245120.

doi:10.1136/bcr-2021-

245120

\section{SUMMARY}

Ankylosis of the temporomandibular joint is a debilitating condition resulting in progressive trismus and facial disfigurement. Common trigger factors include paediatric mandibular trauma, middle ear infection or traumatic childbirth. Although diminishing in incidence among urban population, it is still prevalent in the underprivileged world. Substandard child safety norms, delayed presentation, lack of access to specialties like maxillofacial surgery in rural areas and absence of follow-up contribute to ankylosis. Afflictions in ankylosis are multipronged, involving aesthetic, functional, psychological and nutritional implications to name a few. The damage this pathology causes to the young mind and their morale is humongous. Although well documented, a complete presentation of cases with follow-up till oral and myofascial rehabilitation is seldom reported. This paper describes multidisciplinary management of a young female patient with temporomandibular joint ankylosis. Staged management commencing from interpositional arthroplasty until dental restoration with a 2-year follow-up is presented in this paper.

\section{BACKGROUND}

Temporomandibular joint (TMJ) ankylosis is a punitive disorder predominantly affecting children from the rural or suburban background. The condition involves fusion of the mandibular condyle to the glenoid fossa causing restricted mouth opening and facial disharmony. It is often a delayed complication of mandibular trauma or middle ear infection in early life, which is neglected without adequate follow-up after managing the acute phase. ${ }^{1-3}$ Compromised child safety norms leading to paediatric injuries and uncertain pattern of long-term review that exist in the underdeveloped areas largely contribute to this situation. ${ }^{4}$ Goals of management include achieving mouth opening, restoring facial form and facilitating oral hygiene. ${ }^{5-7}$ But often, patients are either not reporting during the early stages or lost for follow-up once mouth opening is achieved, sidelining the other aspects. The authors in this study have encountered delayed presentation as late as 3-4 decades of living with ankylosis. Treatment protocols for managing TMJ ankylosis are well established in literature depending on the age of presentation, but seldom do they include oral and myofascial rehabilitation. ${ }^{2} 3568-10$ It is of the authors' contention that a protocol be formulated keeping in mind long-term implications and quality of life following treatment of TMJ ankylosis.

\section{CASE PRESENTATION}

A 35-year-old woman from a rural background reported to us with inability to open mouth and an unpleasant facial profile that had developed over the last 25 years (figures $1-3$ ). She was accompanied by her mother who gave a history of apparent ear infection when she was 6 which had been managed using oral medications at the rural peripheral health centre. The current appearance and mouth opening issues has been progressively developing a few years after this incident. The patient's family was undergoing psychological trauma due to alienation by the society considering her condition as a curse. They were unaware that the condition could be treated till recently when a medical camp was conducted in their area.

In spite of nil mouth opening, the patient appeared healthy and fairly nourished. Local examination revealed a typical 'bird facies' appearance with severe mandibular retrusion (convex facial profile, obtuse chin-throat angle. A thorough oral examination was obviously not possible at that moment, although severe crowding and gross carious lesions were evident.

\section{INVESTIGATIONS}

Orthopantomogram was obtained at the preliminary visit. Distorted morphology of the TMJ due to ankylosis was evident bilaterally, including shortened mandibular ramus, prominent antigonial notching and elongated coronoid process. Multiple root stumps and teeth with carious lesions were also meticulously documented for treatment prioritisation once comfortable mouth opening is achieved.

CT scans of $0.3 \mathrm{~mm}$ slices were obtained for analysis and fabrication of skull models. This helped us plan for distraction osteogenesis. Monofocal external pin fixated distractor devices were rehearsed over the skull model (figure 4) and the trial osteotomy was performed.

Airway assessment included volumetric analysis from CT scans and a polysomnography, both of which confirmed diminished upper airway space and obstructive sleep apnoea.

Finally, the standard laboratory workup as for any other elective surgery in an otherwise healthy young adult was ordered. 


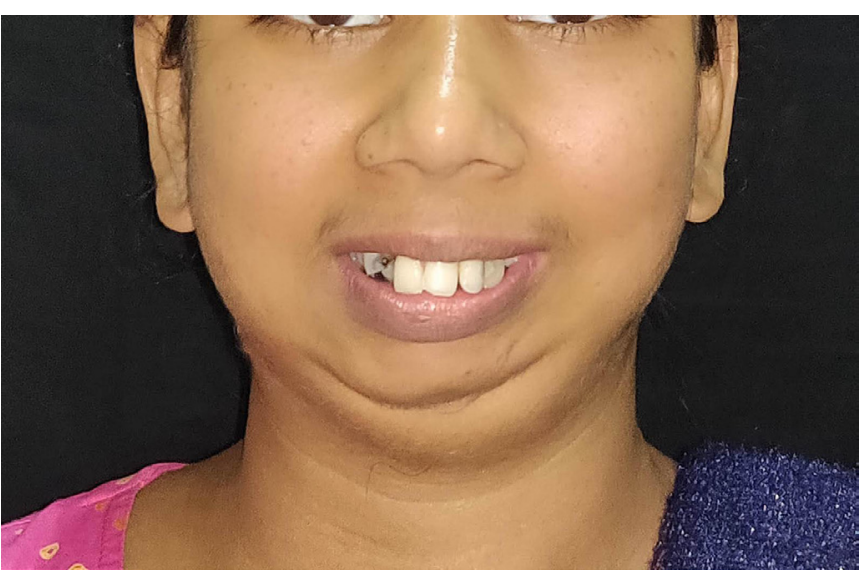

Figure 1 Preoperative frontal view.

\section{TREATMENT}

The surgical problem list included bilaterally ankylosed TMJ, anteroposteriorly deficient mandible and a resultant diminution of upper airway space. Poor oral hygiene due to long-term trismus was evident following limited direct visualisation and orthopantomogram.

The treatment was executed in stages involving interpositional arthroplasty, mandibular distraction osteogenesis, genioplasty and dental and oral myofascial rehabilitation using a combination of implants and fixed partial crowns, bridges and guiding elastics.

Endotracheal intubation was achieved using blind nasal technique with regional anaesthesia. TMJs were exposed using a preauricular approach with temporal extension as described by Al-Kayat and Bramley. ${ }^{11}$ The ankylotic mass was resected along with the coronoid process bilaterally and a mouth opening of $45 \mathrm{~mm}$ was achieved on table (figure 5). A partial thickness temporalis myofascial flap was used as an interpositional material to prevent recurrence (figure 6). Subsequently, mandibular body and angle regions were exposed using submandibular incisions. Osteotomies were performed as planned in the preoperative sessions and monofocal pin-fixated external mandibular distractors were secured (figure 6). Care was taken to prevent any damage to the inferior alveolar neurovascular bundle during osteotomy. The patient was extubated comfortably and recovery

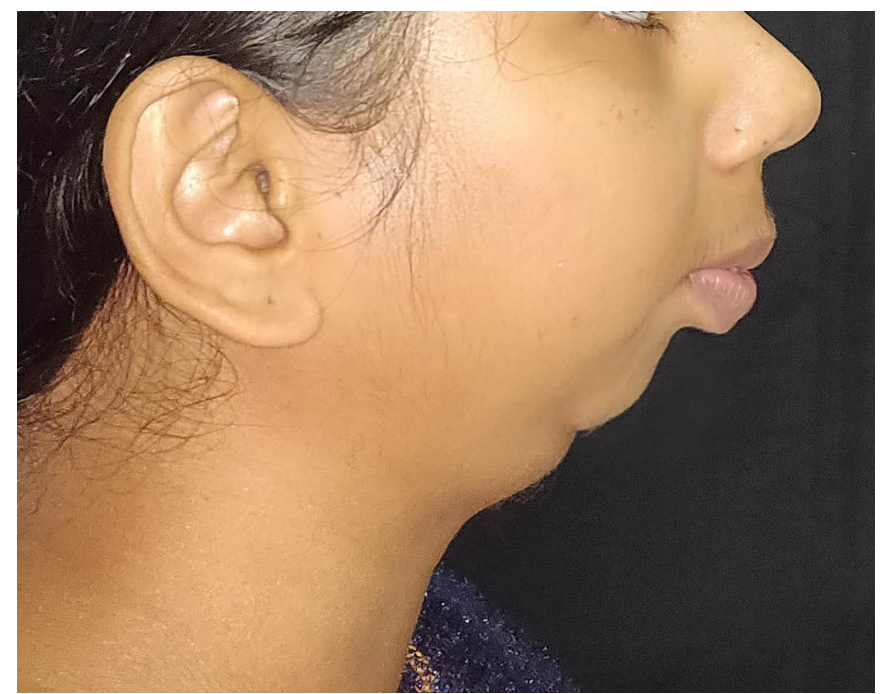

Figure 2 Preoperative side profile view.

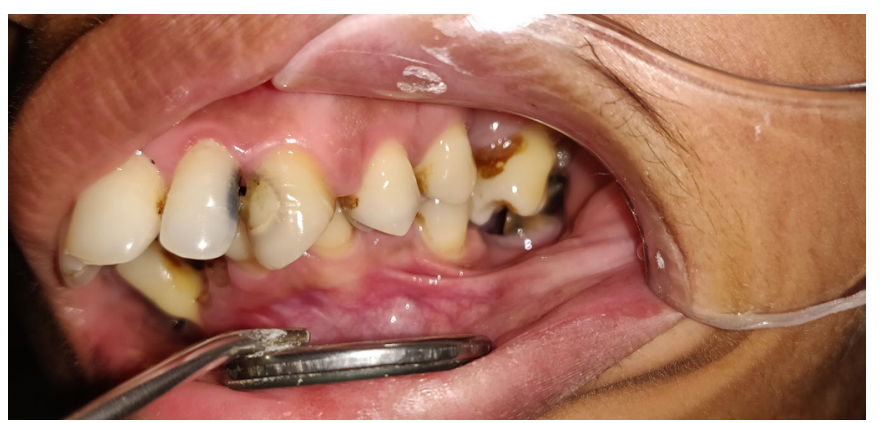

Figure 3 Preoperative dental status, occlusion and mouth opening.

was uneventful. After a latency period of 5 days, distractor devices were activated bilaterally in 12 hourly intervals achieving about $1 \mathrm{~mm}$ of distraction per day. ${ }^{12}$ Mandibular advancement of $25 \mathrm{~mm}$ was achieved by distraction following which a 3-month period was allowed for consolidation of the callus (figure 7). Second stage involved distractor device removal, advancement genioplasty, extraction of teeth with poor prognosis and placement of dental implants (figures 8-10). This was performed together under general anaesthesia. Endotracheal intubation was much easier during this stage due to adequate mouth opening and an advanced mandible as against the initial stage. Prosthodontic phase of the implants and final finishing were completed as outpatient procedures during subsequent visits (figures 11 and 12).

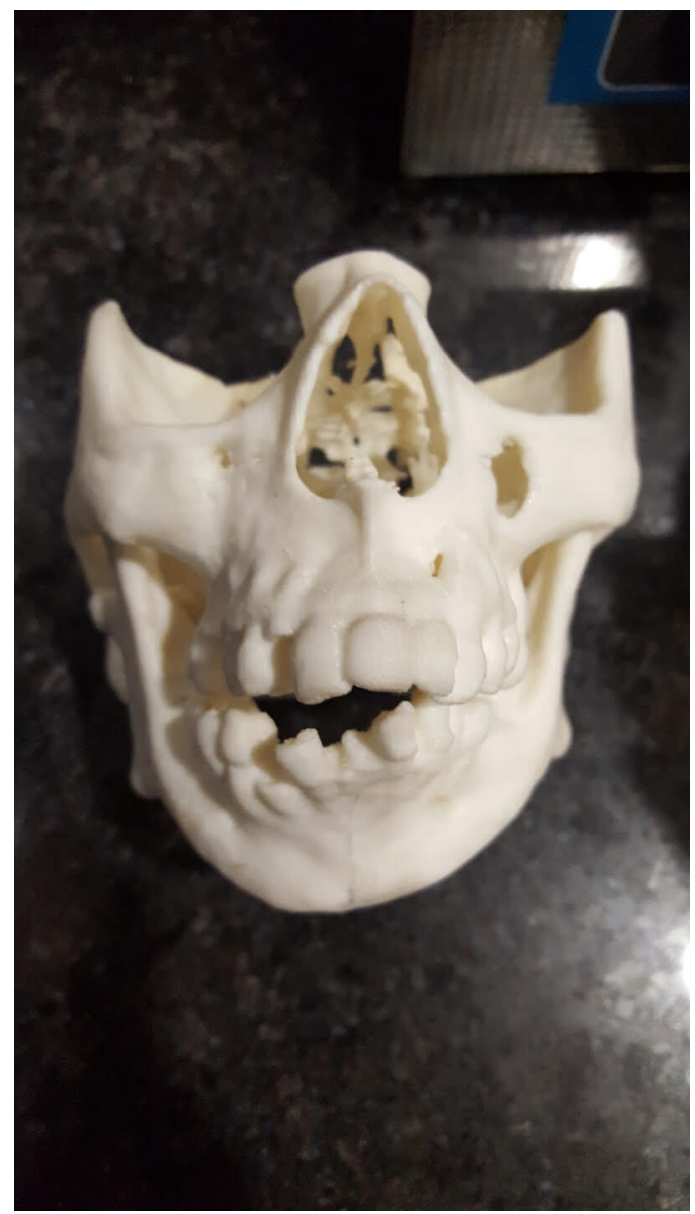

Figure 4 Model for surgical planning. 


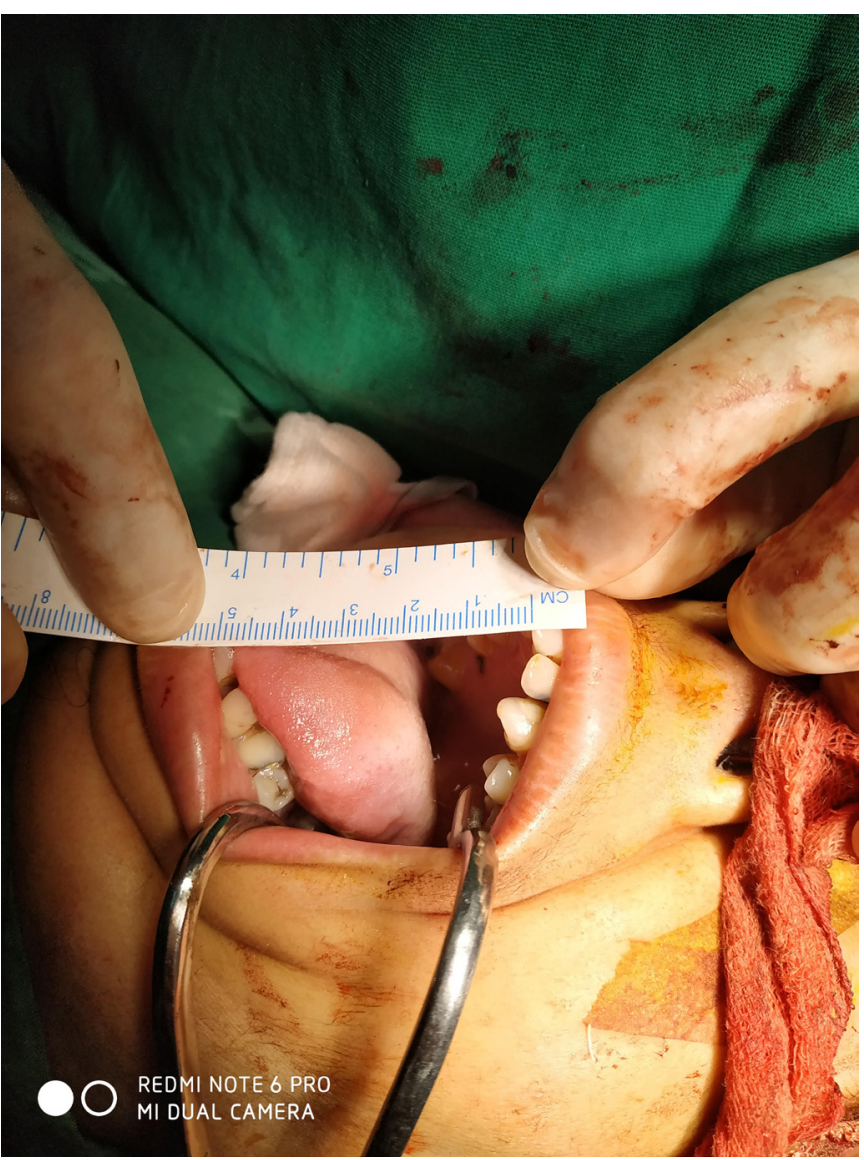

Figure 5 Achievement of $45 \mathrm{~mm}$ mouth opening after release of ankylosis.

\section{OUTCOME AND FOLLOW-UP}

The patient has been on follow-up for 2 years now with optimal mouth opening of $35 \mathrm{~mm}$ (figure 13) and diminished snoring. She is very pleased with her facial appearance and profile (figures 14 and 15). Masticatory efficacy and oral hygiene is good and is being monitored by the dental hygienist (figures 11 and 12).

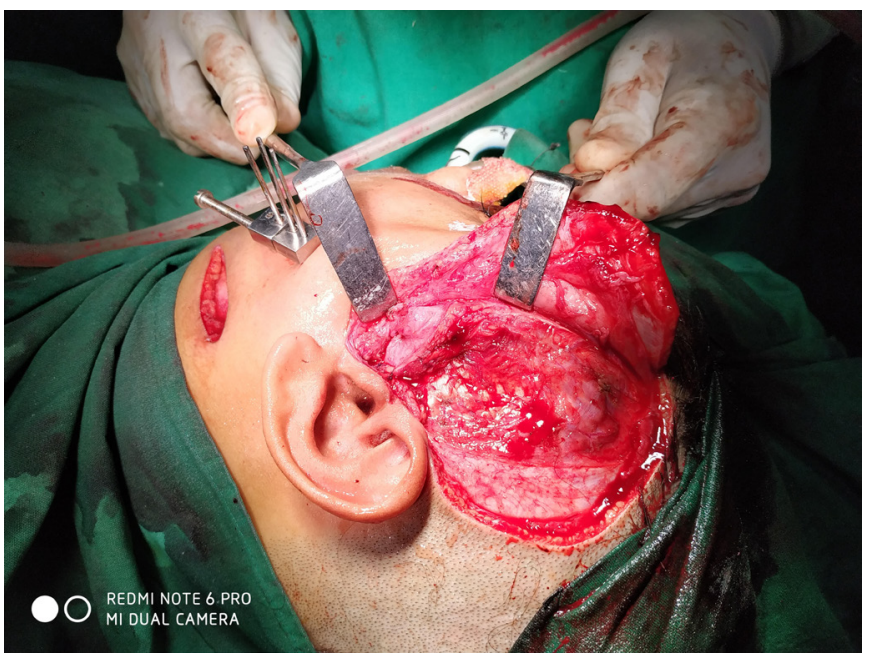

Figure 6 Interposition of temporalis myofascial flap and fixation of mandibular distractor.

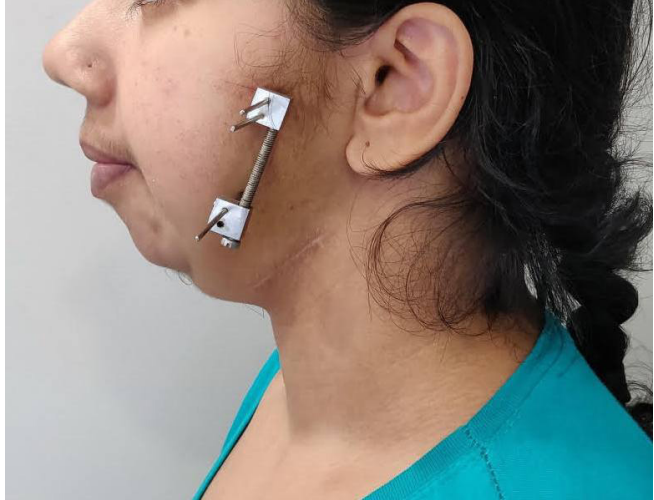

Figure 7 Completion of distraction phase.

\section{DISCUSSION}

TMJ ankylosis is a well-documented entity in regard to trismus and facial deformity. Incidence of ankylosis has reduced in the developed countries but not proportionate to that of the underprivileged world. ${ }^{213}$ Although treatment goals include achieving mouth opening, restoring facial symmetry and offering oral rehabilitation, studies on ankylosis seldom report the final phase of management. Diverse child safety norms, paediatric mandibular trauma, middle ear infection, awareness of maxillofacial surgery as a specialty, multidisciplinary approach, psychosocial impact of facial asymmetry and long term follow-up are the key aspects of ankylosis for detailed discussion in this paper.

It is not uncommon for children to present with acute middle ear infections and the condition is manageable at general practitioners' level with supportive therapy and antibiotic cover. ${ }^{1-3}$ But, worsening mouth opening and diminishing oral intake in a child who has had an episode of middle ear infection in the past is a cause for concern that can be identified predominantly by a dental or maxillofacial surgeon. Although a considerable number of graduates specialise to become maxillofacial surgeons annually, these specialists are a luxurious commodity in rural and suburban areas. This is due to uneven settlement across geographical boundaries and more concentration of surgeons at the urban areas for obvious reasons. Although treated for middle ear infection at the age of 6 and suffering from progressive diminution of mouth opening in the subsequent period, our patient

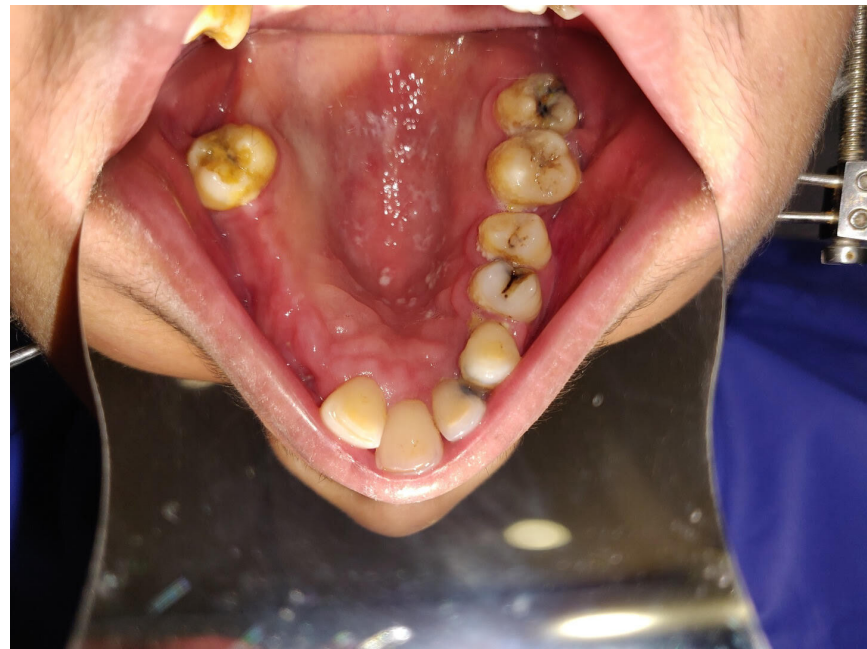

Figure 8 Completed extraction of infection root stumps. 


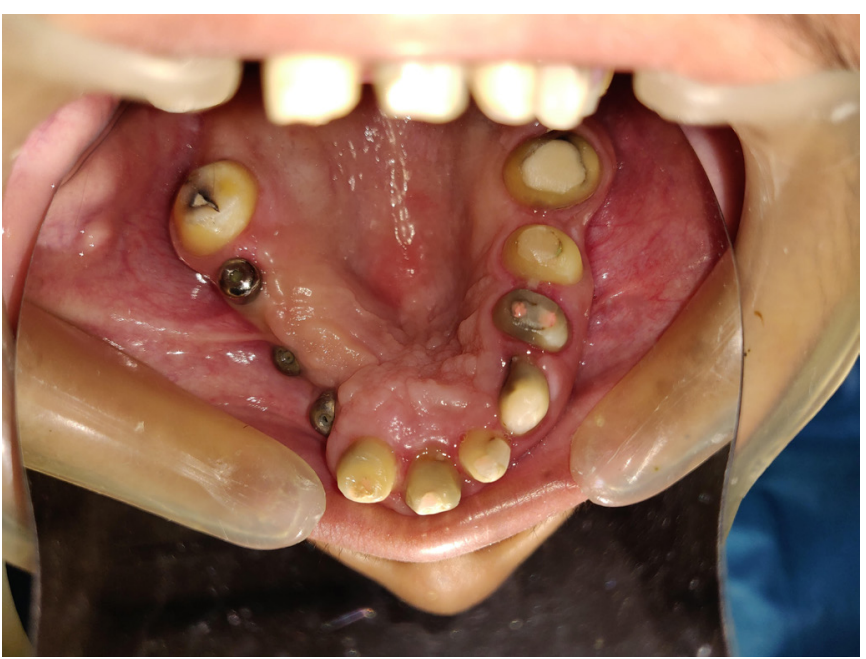

Figure 9 Placement of implants and completion of endodontic therapy for salvageable teeth.

had spent two decades of her life with nil mouth opening before even considering the option of specialist consultation.

Mandibular trauma in early life is another common cause for TMJ ankylosis. Chin trauma results in transmission of kinetic energy towards the richly vascular mandibular condyle causing haemarthrosis. ${ }^{1}$ This unlocks a cascade of osteogenic events leading to uncontrolled bone deposition in the joints resulting in progressive restriction of mouth opening. A child travelling in a two wheeler, especially seated in front of the rider is a common sight in many rural areas. Additionally, it is not universal norm for all passengers to wear helmet and these children take the brunt of impact in case of a road traffic accident. Although such trauma would be received at the regional health centres, it is often the more severe or life-threatening injuries which are attended to and injuries with long-term implications like condylar concussion and haemarthrosis are often neglected.

Preliminary challenge in surgical management of TMJ ankylosis is anaesthesia, due to factors like retrusion of mandible, restriction of pharyngeal space, apparent macroglossia and trismus to name a few. ${ }^{914}$ There are still undocumented instances of ankylosis release under sedation and local anaesthesia to rapidly achieve mouth opening following which the patient gets intubated for completion of the planned procedure. Although

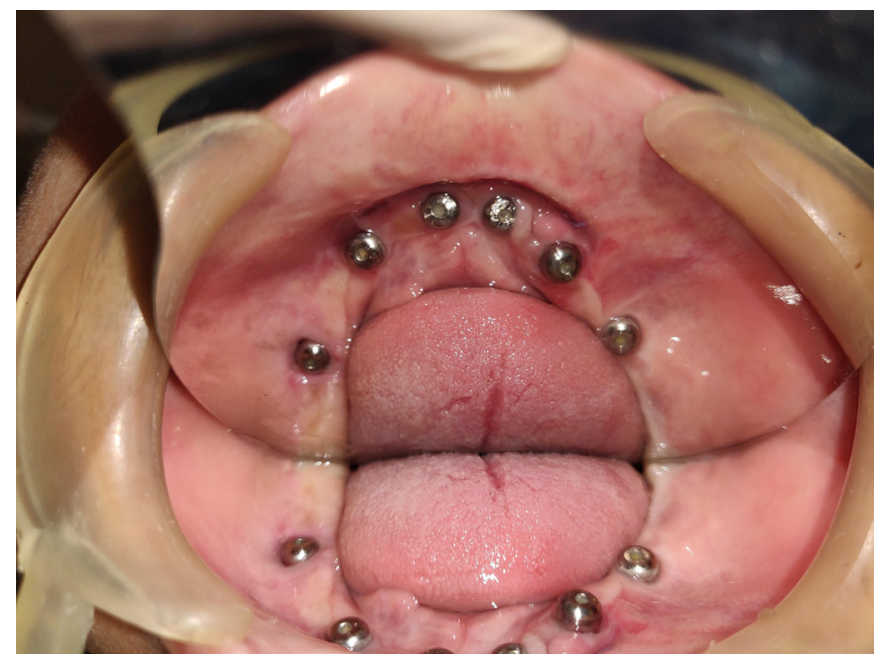

Figure 10 Full arch mandibular implants.

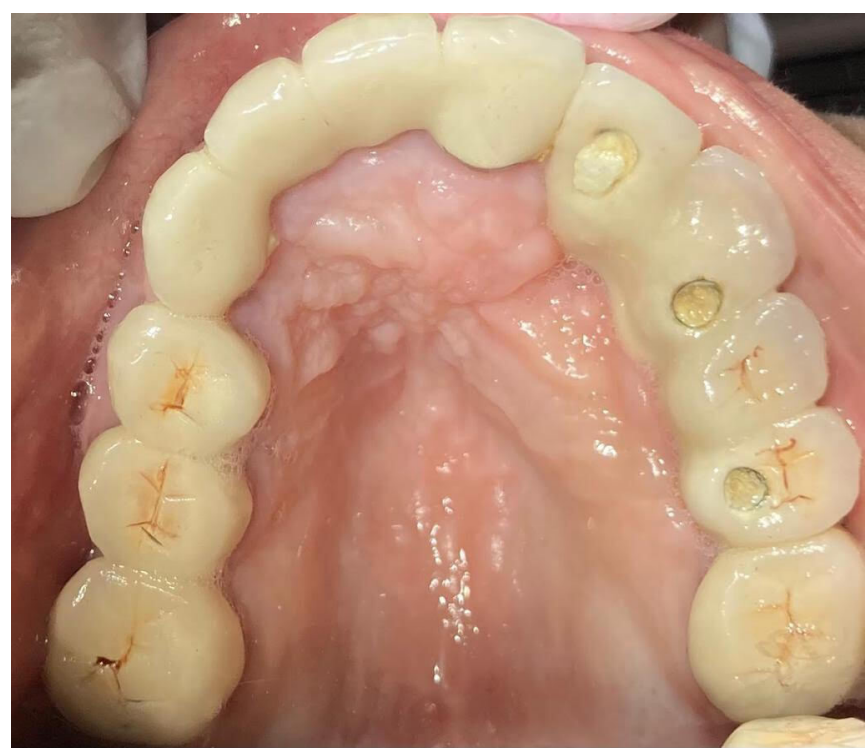

Figure 11 Mandibular dental rehabilitation completed.

primitive, these methods have been justified in setups which are far from being equipped both in terms of distance and time period. Fiberoptic laryngoscope is a luxury for endotracheal intubation in such cases, which our team did not enjoy. Hence, 'awake blind nasal intubation' was performed and patient was positioned for surgery.

Surgical protocol for management of ankylosis is described in detail by Kaban. ${ }^{2}$ Simultaneous release of ankylosis and distraction of the micrognathic mandible is an option proposed to alleviate the need for multiple operative interventions and achieve aesthetic and functional goals in single stage. ${ }^{15} 16$ But as with any other algorithm, the technique has its own disadvantages, which our team was prepared to sideline, considering the socioeconomic situation of our patient. Hence a single stage ankylosis release and mandibular lengthening was performed bilaterally in this case. Distraction osteogenesis involves osteotomy of the mandible and fixation of the distractor device which can be activated after 3-5 days of latency period in the postoperative phase. ${ }^{12}$ Activation is performed on a fixed rate and rhythm to

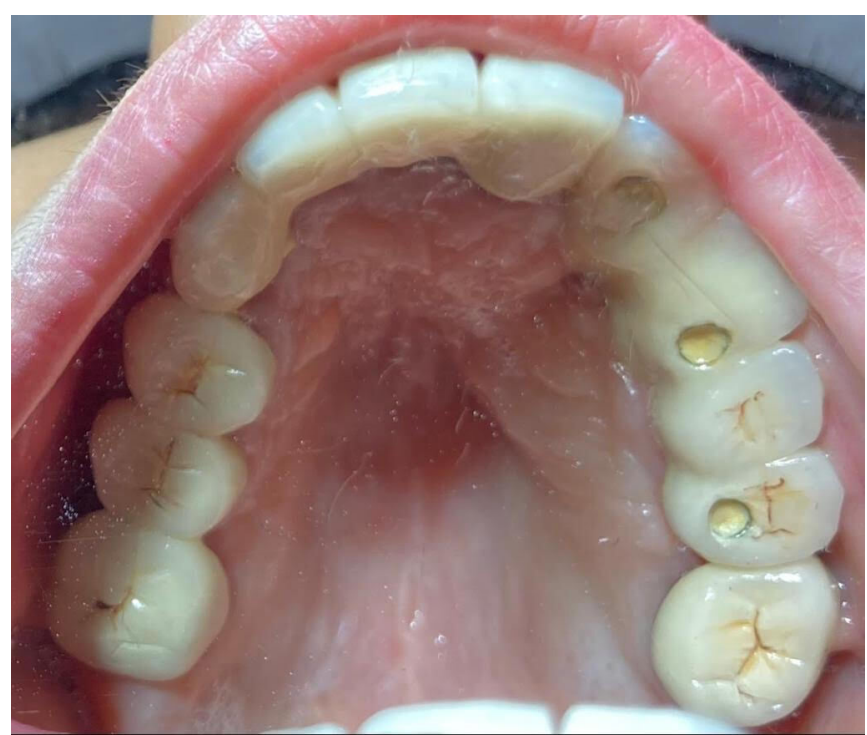

Figure 12 Maxillary dental rehabilitation completed. 


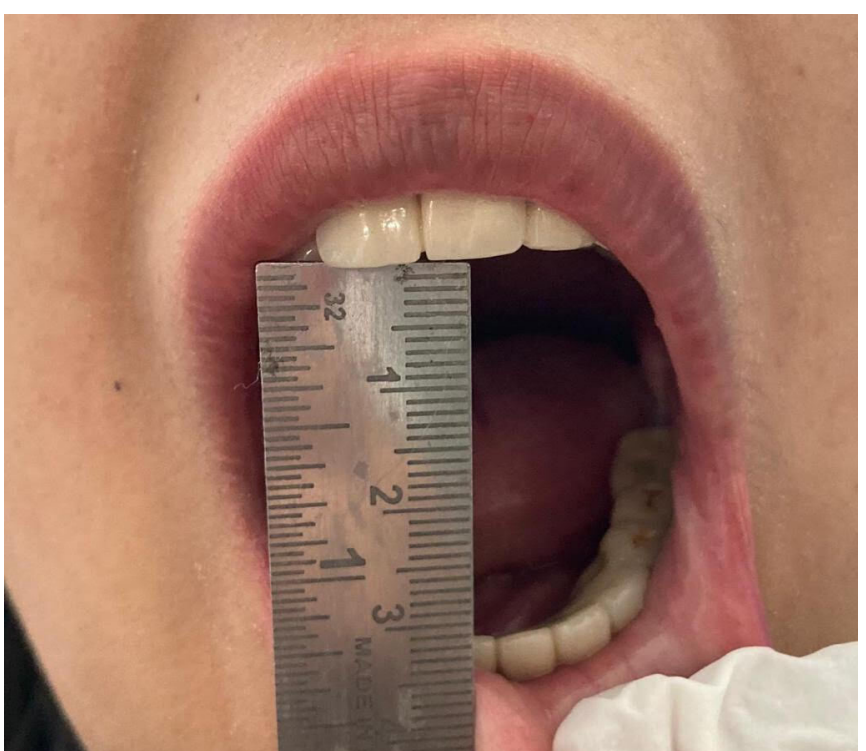

Figure 13 Optimal mouth opening in long term.

achieve lengthening. Once the desired length is achieved, activation is stalled and the elongated bone is allowed to consolidate for a period of 12-16 weeks. Device removal can be planned at the end of this consolidation period. The final position of the mandible after complete distraction will dictate the amount of chin advancement. Hence, genioplasty was performed as a second procedure combined with distractor removal, dental extractions and implants.

Psychosocial aspects must be one of the main considerations when dealing with facial asymmetry, which in the authors' opinion is trilateral for patients suffering with ankylosis. Inability to open the mouth and chew food are as demoralising as having a disfigured face. Masticatory dynamics is only as good as the condition of teeth, periodontium and the alveolar process. With prolonged periods of nil mouth opening as in our patient, it becomes almost impossible to ensure oral health, leading to a deterioration of masticatory efficacy. A comprehensive dental status evaluation was done and a separate plan was charted out for rehabilitation. Accordingly, extraction of multiple root stumps, restoration of the salvageable ones and replacement of missed teeth were completed over the subsequent visits. Restoration of oral environment does not instantly increase full functionality, since it was dormant for two decades. A diet plan was charted out, starting with soft semisolid diet progressing to solid and fibrous food over the next few weeks for periodontal stimulation. ${ }^{17} 18$ Additionally, the myofascial imbalance causes by sudden mouth opening and use of temporalis muscle as a

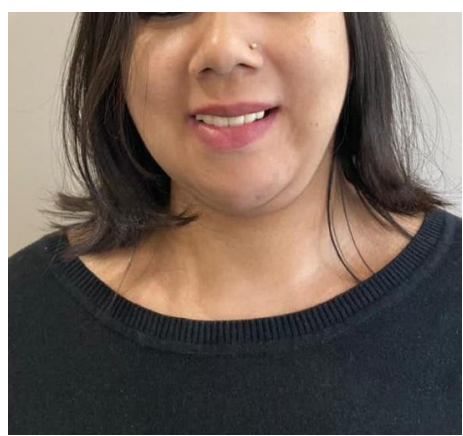

Figure 14 Frontal view at 2-year-follow-up.

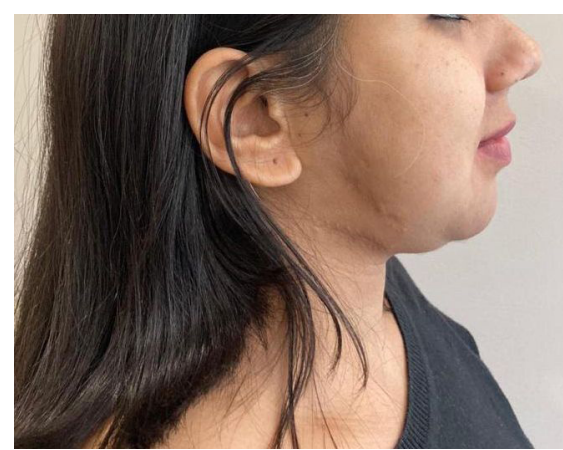

Figure 15 Profile view at 2-year follow-up.

Patient's perspective

'Happy to have regained the ability to consume food of my choice and feel confident to face others at work and off work'.

\section{Learning points}

- Standard child safety norms must become universal policy across geographical boundaries.

- Paediatric maxillofacial surgery should evolve as a superspecialty and become more available in areas of need.

- Treatment for temporomandibular joint ankylosis does not end with mouth opening or facial symmetry. It has to culminate in oral rehabilitation and follow-up.

- Myofascial training of the face is essential for regaining tone of masticatory muscles after surgical treatment for ankylosis.

flap needs to be restored in a similar phased manner. This was completed using masticatory muscle training and intermaxillary guiding elastics.

Successful treatment for ankylosis involves surgical release of ankylosis, restoration of facial form, function and oral health, helping in reinstatement of a confident individual back into the society. The patient reported in this paper landed in a secretarial job and is an ambassador for child safety in her region.

Acknowledgements The authors would like to acknowledge Prof.Subramonian Sivaraj for surgical collaboration

Contributors NJ: conceptualisation, manuscript preparation-writing, reviewing and editing, clinical Support. KK: clinical support, manuscript preparation-reviewing and editing. AR: manuscript preparation-editing.

Funding The authors have not declared a specific grant for this research from any funding agency in the public, commercial or not-for-profit sectors.

Competing interests None declared.

Patient consent for publication Obtained.

Provenance and peer review Not commissioned; externally peer reviewed.

ORCID iD

Naveenkumar Jayakumar http://orcid.org/0000-0001-7636-4247

\section{REFERENCES}

1 Bhatt KV, Roychoudhury A, Bhutia O. Pathogenesis of temporomandibular joint Ankylosis: a perspective. Natl J Maxillofac Surg 2020;11:154-5.

2 Kaban LB, Bouchard C, Troulis MJ. A protocol for management of temporomandibular joint ankylosis in children. J Oral Maxillofac Surg 2009;67:1966-78.

3 Loveless TP, Bjornland T, Dodson TB, et al. Efficacy of temporomandibular joint Ankylosis surgical treatment. J Oral Maxillofac Surg 2010;68:1276-82. 
4 Peden MM, UNICEF WHO, eds. World report on child injury prevention. Geneva, Switzerland: [New York, NY]: World Health Organization; UNICEF, 2008.

5 Andrade NN, Kalra R, Shetye SP. New protocol to prevent TMJ reankylosis and potentially life threatening complications in triad patients. Int I Oral Maxillofac Surg 2012;41:1495-500.

6 el-Sheikh MM, Medra AM. Management of unilateral temporomandibular ankylosis associated with facial asymmetry. J Craniomaxillofac Surg 1997;25:109-15.

7 Seo BY, Eo MY, Park MW. Gap arthroplasty with active mouth opening exercises using an interocclusal splint in temporomandibular joint ankylosis patients | Maxillofacial Plastic and Reconstructive Surgery | Full Text [Internet], 2019. Available: https:// jkamprs.springeropen.com/articles/10.1186/s40902-019-0200-x [Accessed 5 Jun 2021].

8 Andrade NN, Choradia S, Sriram S G. An institutional experience in the management of pediatric mandibular fractures: a study of 74 cases. J Craniomaxillofac Surg 2015;43:995-9

9 Anchlia S, Vyas SM, Dayatar RG, et al. Guidelines for single-stage correction of TMJ Ankylosis, facial asymmetry and OSA in adults. J Maxillofac Oral Surg 2019;18:419-27.

10 Khanna JN, Ramaswami R. Protocol for the management of ankylosis of the temporomandibular joint. Br J Oral Maxillofac Surg 2019;57:1113-8.
11 Al-Kayat A, Bramley P. A modified pre-auricular approach to the temporomandibular joint and malar arch. Br J Oral Surg 1979;17:91-103.

12 McCarthy JG. The role of distraction osteogenesis in the reconstruction of the mandible in unilateral craniofacial microsomia. Clin Plast Surg 1994;21:625-31.

13 Gupta VK, Mehrotra D, Malhotra S, et al. An epidemiological study of temporomandibular joint ankylosis. Natl J Maxillofac Surg . 2012;3:25-30.

14 Jain G, Barik AK, Banerjee A, et al. Airway challenges in bilateral temporomandibular joint ankylosis with adenoid hypertrophy: a case report. J Oral Biol Craniofac Res 2019;9:256-8

15 Munro IR, Chen YR, Park BY. Simultaneous total correction of temporomandibular ankylosis and facial asymmetry. Plast Reconstr Surg 1986;77:517-27.

16 Sharma A, Paeng J-Y, Yamada T, et al. Simultaneous gap arthroplasty and intraoral distraction and secondary contouring surgery for unilateral temporomandibular joint Ankylosis. Maxillofac Plast Reconstr Surg 2016;38:12.

17 Kaur T, Krishnan G, Sharma A. Factors influencing treatment plan and post operative compliance in TMJ Ankylosis patients: a retrospective analysis of long term post surgical evaluation. J Maxillofac Oral Surg 2015;14:17-23.

18 Dimitroulis G. Management of temporomandibular joint disorders: a surgeon's perspective. Aust Dent J 2018;63 Suppl 1:S79-90.

Copyright 2021 BMJ Publishing Group. All rights reserved. For permission to reuse any of this content visit https://www.bmj.com/company/products-services/rights-and-licensing/permissions/

BMJ Case Report Fellows may re-use this article for personal use and teaching without any further permission.

Become a Fellow of BMJ Case Reports today and you can:

- Submit as many cases as you like

- Enjoy fast sympathetic peer review and rapid publication of accepted articles

Access all the published articles

- Re-use any of the published material for personal use and teaching without further permission

\section{Customer Service}

If you have any further queries about your subscription, please contact our customer services team on +44 (0) 2071111105 or via email at support@bmj.com.

Visit casereports.bmj.com for more articles like this and to become a Fellow 